\title{
Electromagnetic splitting of quark and pseudoscalar meson masses from dynamical QCD + QED
}

\section{R. Horsley ${ }^{a}$, Y. Nakamura ${ }^{b}$, D. Pleiter ${ }^{c}$, P.E.L. Rakow ${ }^{d}$, G. Schierholz ${ }^{* e}$, H. Stüben ${ }^{f}$, R.D. Young ${ }^{g}$ and J.M. Zanotti ${ }^{g}$}

${ }^{a}$ School of Physics and Astronomy, University of Edinburgh, Edinburgh EH9 3JZ, United Kingdom

${ }^{b}$ RIKEN Advanced Institute for Computational Science, Kobe, Hyogo 650-0047, Japan

c JSC, Forschungszentrum Jülich, 52425 Jülich, Germany

${ }^{d}$ Theoretical Physics Division, Department of Mathematical Sciences, University of Liverpool, Liverpool L69 3BX, United Kingdom

${ }^{e}$ Deutsches Elektronen-Synchrotron DESY, 22603 Hamburg, Germany

${ }^{f}$ RRZ, University of Hamburg, 20146 Hamburg, Germany

${ }^{g}$ CSSM, School of Chemistry and Physics, University of Adelaide, Adelaide SA 5005, Australia

\section{QCDSF Collaboration}

Lattice QCD simulations are now reaching a precision where electromagnetic corrections from QED become important. In investigating the effects of SU(3) breaking due to quark mass differences within QCD, a group-theoretical analysis of the mass dependence greatly helped us organize our results. We now do the same with electromagnetic charge effects by extending the calculations to dynamical $1+1+1$ flavor QCD + QED.

The XXXI International Symposium on Lattice Field Theory

July 29 - August 3, 2013

Mainz, Germany

\footnotetext{
* Speaker.
} 


\section{Introduction}

One of the most profound open questions in particle physics is to understand the pattern of flavor symmetry breaking and mixing, and the origin of CP violation. In [1] we have outlined a program to systematically investigate the pattern of flavor symmetry breaking. The program has been successfully applied to meson and baryon masses involving up $(u)$, down $(d)$ and strange $(s)$ quarks.

A distinctive feature of our simulations is the way we tune the light and strange quark masses. We have our best theoretical understanding when all three quark flavors have the same mass, because we can use the full power of flavor $\mathrm{SU}(3)$. Starting from the $\mathrm{SU}(3)$ symmetric point, our strategy is to keep the singlet quark mass $\bar{m}=\left(m_{u}+m_{d}+m_{s}\right) / 3$ fixed at its physical value, while $\delta m_{q} \equiv m_{q}-\bar{m}, q=u, d, s$ is varied. As we move from the symmetric point $m_{u}=m_{d}=m_{s}$ (where the pion mass is $\sim 411 \mathrm{MeV}$ ) to the physical point along the path $\bar{m}=$ constant, the $s$ quark becomes heavier, while the $u$ and $d$ quarks become lighter. These two effects tend to cancel in any flavor singlet quantity. To leading order, the cancellation is exact at the symmetric point, and we have found that it remains good down to the lightest points we have simulated so far [1].

In order to compute physical observables to high precision, it is important to include and control contributions from QED. Recent lattice investigations of electromagnetic (EM) corrections to hadron observables have been performed on pure QCD background configurations [2], while a simulation with dynamical photons, including meson-photon mixing effects, is still missing. In this project we will extend our previous simulations of $2+1$ flavor QCD with SLiNC fermions to a fully dynamical simulation of $1+1+1$ flavor QCD + QED.

\section{QCD + QED pseudoscalar meson mass formulae}

In pure QCD [1] our strategy was to start from a point with all three sea quark masses equal, $m_{u}=m_{d}=m_{s}$, and extrapolate towards the physical point by keeping the average sea quark mass $\bar{m}=\left(m_{u}+m_{d}+m_{s}\right) / 3$ constant. For this trajectory to reach the physical point we start at a point $\bar{m}=m_{0}$, where $M_{\pi}=M_{K}$ with $2 M_{K}^{2}+M_{\pi}^{2}$ equal to its physical value. That is $M_{\pi}=M_{K}=413 \mathrm{MeV}$. We call this point the physical SU(3) symmetric point. We denote the distance from $m_{0}$ by $\delta m_{q}=$ $m_{q}-m_{0}, q=u, d, s$. This forms a plane, as we have the constraint $\delta m_{u}+\delta m_{d}+\delta m_{s}=0$. The bare quark masses are defined by

$$
a m_{0}=\frac{1}{2 \kappa_{0}}-\frac{1}{2 \kappa_{c}}, \quad a m_{q}=\frac{1}{2 \kappa_{q}}-\frac{1}{2 \kappa_{c}},
$$

where $\kappa_{0}$ gives the quark mass at the physical SU(3) symmetric point, and where vanishing of all quark masses along the line $\kappa_{u}=\kappa_{d}=\kappa_{s}$ determines $\kappa_{c}$. The quark masses $m_{q}$ are subject to additive and multiplicative renormalization, while the reference point $m_{0}$ gets multiplicatively renormalized only [1].

In this presentation we shall concentrate on the pseudoscalar meson octet. The expansion around $m_{q}=m_{0}$, valid for the outer ring of the pseudoscalar octet, was found to be [1]

$$
\begin{aligned}
M^{2}(a \bar{b}) & =M_{0}^{2}+\alpha\left(\delta m_{a}+\delta m_{b}\right) \\
& +\beta_{0} \frac{1}{6}\left(\delta m_{u}^{2}+\delta m_{d}^{2}+\delta m_{s}^{2}\right)+\beta_{1}\left(\delta m_{a}^{2}+\delta m_{b}^{2}\right)+\beta_{2}\left(\delta m_{a}-\delta m_{b}\right)^{2}
\end{aligned}
$$


for arbitrary quarks $q=a, b$, with $\alpha$ and $\beta_{0}, \beta_{1}, \beta_{2}$ being the LO and NLO expansion coefficients, respectively.

It is useful, in many respects, to vary valence and sea quark masses independently. This is referred to as partial quenching (PQ). In this case the sea quark masses remain constrained by $\bar{m}=$ constant, while the valence quark masses $\mu_{u}, \mu_{d}, \mu_{s}$ are unconstrained. Defining $\delta \mu_{q}=\mu_{q}-\bar{m}$, we obtain the PQ mass formula

$$
\begin{aligned}
M^{2}(a \bar{b}) & =M_{0}^{2}+\alpha\left(\delta \mu_{a}+\delta \mu_{b}\right) \\
& +\beta_{0} \frac{1}{6}\left(\delta m_{u}^{2}+\delta m_{d}^{2}+\delta m_{s}^{2}\right)+\beta_{1}\left(\delta \mu_{a}^{2}+\delta \mu_{b}^{2}\right)+\beta_{2}\left(\delta \mu_{a}-\delta \mu_{b}\right)^{2}
\end{aligned}
$$

When $\mu_{q} \rightarrow m_{q}$, this result reduces to the previous result (2.2). The coefficients that appear in the expansion about the flavor symmetric point (2.2) and in the PQ case (2.3) are the same. Hence this offers a computationally cheaper way of obtaining them.

The symmetry of the electromagnetic current is similar to the symmetry of the quark mass matrix. The simplifications that come from the constraint $\delta m_{u}+\delta m_{d}+\delta m_{s}=0$ in the mass case are similar to the simplifications we get from the identity $e_{u}+e_{d}+e_{s}=0$. One difference between quark mass and electromagnetic expansions is that in the mass expansion we can have both odd and even powers of $\delta m_{q}$, whereas we are only allowed even powers of the quark charges. We can therefore read off the leading QED corrections from [1], dropping the linear terms and changing masses to charges. For the outer mesons, and also for the partially quenched $q \bar{q}$ mesons with all annihilation diagrams turned off, we find

$$
\begin{aligned}
M^{2}(a \bar{b}) & =M_{0}^{2}+\alpha\left(\delta \mu_{a}+\delta \mu_{b}\right) \\
& +\beta_{0} \frac{1}{6}\left(\delta m_{u}^{2}+\delta m_{d}^{2}+\delta m_{s}^{2}\right)+\beta_{1}\left(\delta \mu_{a}^{2}+\delta \mu_{b}^{2}\right)+\beta_{2}\left(\delta \mu_{a}-\delta \mu_{b}\right)^{2} \\
& +\beta_{0}^{\mathrm{EM}}\left(e_{u}^{2}+e_{d}^{2}+e_{s}^{2}\right)+\beta_{1}^{\mathrm{EM}}\left(e_{a}^{2}+e_{b}^{2}\right)+\beta_{2}^{\mathrm{EM}}\left(e_{a}-e_{b}\right)^{2} \\
& +\gamma_{0}^{\mathrm{EM}}\left(e_{u}^{2} \delta m_{u}+e_{d}^{2} \delta m_{d}+e_{s}^{2} \delta m_{s}\right)+\gamma_{1}^{\mathrm{EM}}\left(e_{a}^{2} \delta \mu_{a}+e_{b}^{2} \delta \mu_{b}\right) \\
& +\gamma_{2}^{\mathrm{EM}}\left(e_{a}-e_{b}\right)^{2}\left(\delta \mu_{a}+\delta \mu_{b}\right)+\gamma_{3}^{\mathrm{EM}}\left(e_{a}^{2}-e_{b}^{2}\right)\left(\delta \mu_{a}-\delta \mu_{b}\right) .
\end{aligned}
$$

The coefficients in (2.4) can be matched up with different classes of Feynman diagrams shown in Fig. 1. The first diagram, with both ends of the photon attached to the same valence quark, contributes to $\left(\beta_{1}^{\mathrm{EM}}+\beta_{2}^{\mathrm{EM}}\right)$ as well as $\left(\gamma_{1}^{\mathrm{EM}}+\gamma_{2}^{\mathrm{EM}}+\gamma_{3}^{\mathrm{EM}}\right)$. The second diagram, with the photon crossing between the valence lines, only contributes to $\beta_{2}^{\mathrm{EM}}$ and $\gamma_{2}^{\mathrm{EM}}$. The last diagram, with the
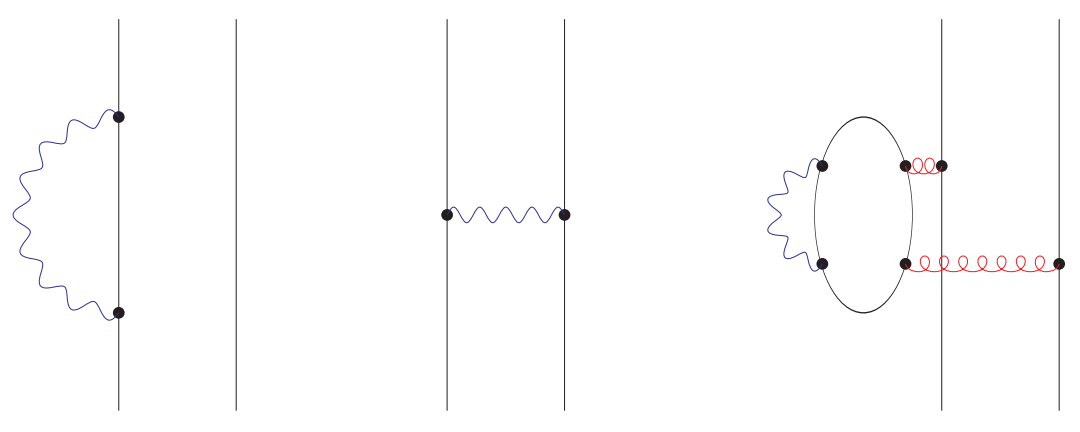

Figure 1: Examples of Feynman diagrams contributing to the meson electromagnetic mass to order $e^{2}$. 
photon being attached to the sea quarks, is an example of a diagram contributing to $\beta_{0}^{\mathrm{EM}}$ and $\gamma_{0}^{\mathrm{EM}}$. It would be missed out if the electromagnetic field was quenched instead of dynamical.

Except for $\beta_{0}, \beta_{0}^{\mathrm{EM}}$ and $\gamma_{0}^{\mathrm{EM}}$, all coefficients can be determined by PQ simulations at our expansion point. The term $\beta_{0}^{\mathrm{EM}}\left(e_{u}^{2}+e_{d}^{2}+e_{s}^{2}\right)$ can be absorbed into $M_{0}^{2}$. The coefficients $\beta_{0}$ and $\gamma_{0}^{\mathrm{EM}}$ require simulations with unequal sea quark masses. Many of the terms in (2.4) cancel in the combination

$$
\begin{aligned}
M^{2}(a \bar{b})-\left[M^{2}(a \bar{a})\right. & \left.+M^{2}(b \bar{b})\right] / 2=\beta_{2}\left(\delta \mu_{a}-\delta \mu_{b}\right)^{2}+\beta_{2}^{\mathrm{EM}}\left(e_{a}-e_{b}\right)^{2} \\
& +\gamma_{2}^{\mathrm{EM}}\left(e_{a}-e_{b}\right)^{2}\left(\delta \mu_{a}+\delta \mu_{b}\right)+\gamma_{3}^{\mathrm{EM}}\left(e_{a}^{2}-e_{b}^{2}\right)\left(\delta \mu_{a}-\delta \mu_{b}\right)
\end{aligned}
$$

that will be important in our later discussions.

\section{Lattice setup}

The action we are using is

$$
S=S_{G}+S_{A}+S_{F}^{u}+S_{F}^{d}+S_{F}^{s} .
$$

Here $S_{G}$ is the tree-level Symanzik improved $\mathrm{SU}(3)$ gauge action, and $S_{A}$ is the noncompact U(1) gauge action [3] of the photon,

$$
S_{A}=\frac{1}{2 e^{2}} \sum_{x, \mu<v}\left(A_{\mu}(x)+A_{v}(x+\mu)-A_{\mu}(x+v)-A_{v}(x)\right)^{2} .
$$

The fermion action for each flavor is

$$
\begin{array}{r}
\tilde{S}_{F}^{q}=\sum_{x}\left\{\frac{1}{2} \sum_{\mu}\left[\bar{q}(x)\left(\gamma_{\mu}-1\right) e^{-i e_{q} A_{\mu}(x)} \tilde{U}_{\mu}(x) q(x+\hat{\mu})-\bar{q}(x)\left(\gamma_{\mu}+1\right) e^{i e_{q} A_{\mu}(x)} \tilde{U}_{\mu}^{\dagger}(x-\hat{\mu}) q(x-\hat{\mu})\right]\right. \\
\left.+\frac{1}{2 \kappa_{q}} \bar{q}(x) q(x)-\frac{1}{4} c_{S W} \sum_{\mu \nu} \bar{q}(x) \sigma_{\mu \nu} F_{\mu \nu}(x) q(x)\right\}
\end{array}
$$

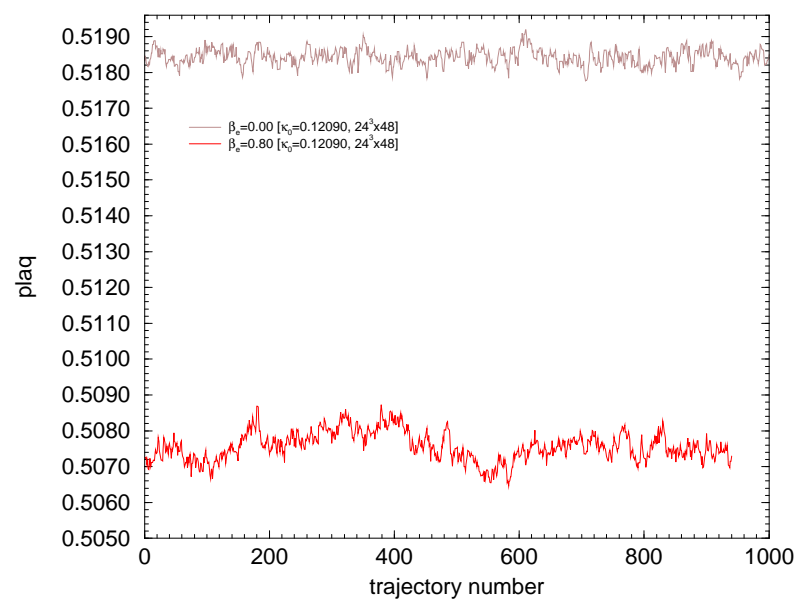

Figure 2: The average plaquette for $\beta=5.50$ and $\kappa_{u}=\kappa_{d}=\kappa_{s}=0.12090$ on the $24^{3} \times 48$ lattice for $e^{2}=1.25$ (bottom red line) and $e^{2}=0$ (top gray line) as a function of trajectory number. 
where $\tilde{U}_{\mu}$ is a single iterated mild stout smeared link [1]. The clover coefficient $c_{S W}$ has been computed nonperturbatively [4]. The quark charges are $e_{u}=2 / 3$ and $e_{d}=e_{s}=-1 / 3$ (in units of $e)$. We presently neglect EM modifications to the clover term. This will leave us with corrections of $O\left(e^{2} a\right)$, which are presumably smaller than the $O\left(a^{2}\right)$ corrections from QCD.

Upon integrating out the Grassmann variables in the partition function, and rewriting the resultant determinant using pseudofermions, the effective action reads (generically)

$$
\begin{aligned}
S\left[U, A,\left\{\phi^{\dagger}, \phi\right\}\right] & =S_{G}[U]+S_{A}[A]+\phi_{u}^{\dagger}\left[\mathscr{M}\left(\kappa_{u}\right)^{\dagger} \mathscr{M}\left(\kappa_{u}\right)\right]^{-\frac{1}{2}} \phi_{u} \\
& +\phi_{d}^{\dagger}\left[\mathscr{M}\left(\kappa_{d}\right)^{\dagger} \mathscr{M}\left(\kappa_{d}\right)\right]^{-\frac{1}{2}} \phi_{d}+\phi_{s}^{\dagger}\left[\mathscr{M}\left(\kappa_{s}\right)^{\dagger} \mathscr{M}\left(\kappa_{s}\right)\right]^{-\frac{1}{2}} \phi_{s},
\end{aligned}
$$

where $\mathscr{M}$ is the fermion matrix. We deal with the square root of $\mathscr{M}^{\dagger} \mathscr{M}$ by rewriting it as a rational function

$$
X^{-1 / n}=\alpha_{0}+\sum_{k=1}^{N} \frac{\alpha_{k}}{X+\beta_{k}}
$$

and employ the Rational Hybrid Monte Carlo (RHMC) algorithm[5]. At the symmetric point, $\kappa_{u}=\kappa_{d}=\kappa_{s}$, this reduces to $2+1$ quark species. Then the Hybrid Monte Carlo (HMC) algorithm can be used for the $d$ and $s$ quarks, while the RHMC algorithm is used for the $u$ quark. Away from the symmetric point we would not expect to run into a sign problem as we will always keep $m_{u} \approx m_{d}$.

\section{Preliminary results and discussion}

Our first dynamical QCD + QED simulation was done on the $24^{3} \times 48$ lattice at $\beta=5.50$ and $\kappa_{u}=\kappa_{d}=\kappa_{s}=0.12090$. That is at the flavor symmetric point $\delta m_{u}=\delta m_{d}=\delta m_{s}=0$. We chose $e^{2}=1.25$. In Fig. 2 we compare the average plaquette with and without dynamical photons. The difference is significant. Our strategy is to simulate at an artificially large coupling $\alpha_{\mathrm{EM}} \approx 1 / 10$, and then interpolate between this point and pure QCD to the physical coupling $\alpha_{\mathrm{EM}}=1 / 137$.

As a first application we have looked at the EM mass shifts of quark and pseudoscalar meson masses. In Fig. 3 we show PQ masses $a M(q \bar{q})$ for $q \bar{q}=u \bar{u}, d \bar{d}(=s \bar{s})$ and a fictitious electrically neutral quark $n, q \bar{q}=n \bar{n}$, as a function of the $\mathrm{PQ}$ hopping parameter $\kappa_{\mathrm{PQ}}$. The first point to notice is that the mesons have become much heavier, especially the $u \bar{u}$. We attribute this mostly to a shift in $\kappa_{c}$ for the quarks, due to their electromagnetic self-interaction, which amounts to an additive quark mass renormalization. We would obviously expect this to be a bigger effect for the $u$ than for the $d$ or $s$ quark, as observed. At the flavor symmetric point, $\kappa_{\mathrm{PQ}}=0.1209$, we find

$$
\begin{array}{ll}
a M(n \bar{n})=0.4606(30), & a M(d \bar{d})=0.5655(16), \\
a M(u \bar{d})=0.7310(15), & a M(u \bar{u})=0.8283(11) .
\end{array}
$$

This is to be compared with the corresponding mass of pure QCD, $a M=0.1779(6)$ [1]. We estimate the lattice spacing $a$ to be $\approx 10 \%$ smaller than in pure QCD, using the vector meson mass for determining the change in scale.

A reasonable definition of the additive quark mass renormalization for each flavor is

$$
\Delta a m_{q}=\frac{1}{2 \kappa_{c}}-\frac{1}{2 \kappa_{c}^{q} \mathrm{PQ}},
$$




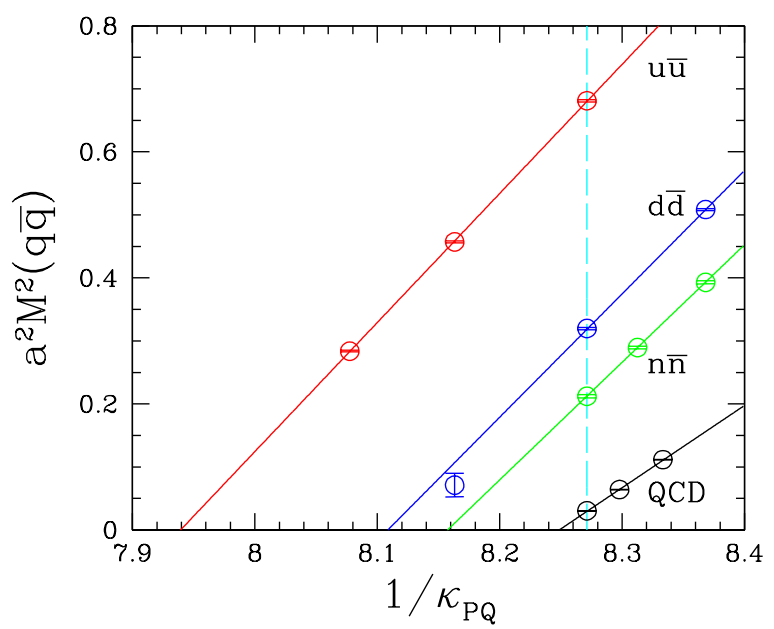

Figure 3: Partially quenched QCD + QED pseudoscalar meson masses $a M(q \bar{q})$ for $q \bar{q}=u \bar{u}, d \bar{d}$ and $n \bar{n}$ against $1 / \kappa_{\mathrm{PQ}}$. Also shown are the PQ masses from pure $\mathrm{QCD}$, as given in [6].

where $\kappa_{c}=0.121252$ is the critical hopping parameter of QCD, and the PQ critical hopping parameter $\kappa_{c \mathrm{PQ}}^{q}$ can be read off from Fig. 3. We find

$$
\Delta a m_{n}=0.036 e^{2}, \quad \Delta a m_{d}=\Delta a m_{s}=0.056 e^{2}, \quad \Delta a m_{u}=0.122 e^{2} .
$$

This is to be compared with the quark mass of pure QCD at the flavor symmetric point, $a m_{u}=$ $a m_{d}=a m_{s}=0.012$. Note that $\left(a m_{u}-a m_{n}\right):\left(a m_{d}-a m_{n}\right) \approx 4: 1$, as expected.

Our present fits give $a^{2} \beta_{0}^{\mathrm{EM}}=1.20 e^{2}$ and $a^{2} \beta_{1}^{\mathrm{EM}}=0.44 e^{2}$, assuming a linear dependence on $e^{2}$. Both $\beta_{0}^{\mathrm{EM}}$ and $\beta_{1}^{\mathrm{EM}}$ come almost entirely from the shifts in $\kappa_{c}$. From PCAC and the leading flavor expansion we expect that $M^{2}(u \bar{d})-\left[M^{2}(u \bar{u})+M^{2}(d \bar{d}] / 2=0\right.$. Violations of this relation cannot be present at leading order in the quark mass. In our data the $\beta_{0}^{\mathrm{EM}}$ and $\beta_{1}^{\mathrm{EM}}$ terms cancel, and the only term which contributes at the expansion point $\delta \mu_{u}=\delta \mu_{d}=\delta \mu_{s}=0$ is $\beta_{2}^{\mathrm{EM}}$,

$$
M^{2}(u \bar{d})-\left[M^{2}(u \bar{u})+M^{2}(d \bar{d}] / 2=\beta_{2}^{\mathrm{EM}},\right.
$$

corresponding to the middle diagram in Fig. 1. From our fits we obtain $a^{2} \beta_{2}^{\mathrm{EM}}=+0.025 e^{2}$.

In order to understand the sign of $\beta_{2}^{\mathrm{EM}}$, we note that opposite charges attract and like charges repel. As a result, we would expect EM effects to raise the mass of, for example, the $u \bar{d}\left(\pi^{+}\right)$meson relative to the $u \bar{u}$ and $d \bar{d}$ mesons. That is exactly what we find, which is mirrored in a positive sign of $\beta_{2}^{\mathrm{EM}}$. We should, however, be aware that this result might be contaminated by QCD and heavy quark effects.

Besides the mass splittings of mesons and baryons, we are interested in the masses of $u, d$ and $s$ quarks. A point to make is that the renormalization factors will now depend on both the QCD and the QED coupling, and the $u$ quark will have a different renormalization factor and anomalous dimension from the other two quarks. This means that the ratio $m_{d} / m_{u}$ now depends on renormalization scheme and scale. Likewise, isospin-violating mass splittings, such as $M_{n}-M_{p}$, 
are scheme independent, but the question of how much of the splitting is due to the quark mass differences, and how much is due to EM effects, becomes dependent on scheme and scale.

\section{Outlook}

In pure QCD we can impose perfect $\mathrm{SU}(3)$ symmetry by making all three $\kappa$ values equal. With QED present, there is no way to have perfect SU(3) symmetry with physical charge ratios. A physically reasonable definition is to look for a line, where the neutral pseudoscalar masses $M(s \bar{d})$, $M(d \bar{s})$ as well as the PQ flavor diagonal masses $M(u \bar{u}), M(d \bar{d}), M(s \bar{s})$ (with annihilation diagrams turned off) and $M(n \bar{n})$ are equal. We are currently using PQ calculations to locate this line. The line will have $\kappa_{d}=\kappa_{s} \neq \kappa_{u}$.

This symmetric line will end at a point, where all neutral pseudoscalar mesons are massless. We define this to be the chiral point. It is the point, where all our quark masses are zero. In the case of the $d$ and $u$ quarks this is the correct definition. Even with QED present, we have a chiral SU(2) symmetry connecting $d$ and $s$ quarks. So, if both quarks are massless, there will be a massless Goldstone boson from the spontaneous symmetry breaking. Although the neutral pseudoscalar mesons will be massless at the chiral point, the charged mesons can have a mass from EM effects. Furthermore, the charged axial vector currents are no longer conserved after QED is added to the action. Hence, there is no Goldstone boson for the charged pseudoscalar sector.

To summarize, our strategy is to compute hadron observables, both in QCD (which we have done already) and in QCD + QED with $\bar{m}$, the average sea quark mass, to be the same (or nearly the same) in both simulations. This we achieve by simulating at points, where the QCD + QED pseudoscalar mesons have (approximately) the same mass as in the pure QCD simulation. To obtain statistically significant results, the calculations are performed at a suitable value of $e^{2}$. We then may interpolate the numbers to $\alpha_{\mathrm{EM}}=1 / 137$, knowing the results at $e^{2}=0$.

\section{Acknowledgement}

This work has been supported in part by the EU under contract 227431 (HadronPhysics2) and contract 283286 (HadronPhysics3), and the Australian Research Council by grants FT120100821 (RDY) and FT100100005 (JMZ). The numerical simulations have been performed on JUQUEEN at JSC (Jülich) and DIRAC 2 at EPCC (Edinbugh), as well as on ICE at HLRN (Berlin and Hannover).

\section{References}

[1] W. Bietenholz et al., Phys. Rev. D 84, 054509 (2011) [arXiv:1102.5300 [hep-lat]].

[2] T. Blum et al., Phys. Rev. D 82 (2010) 094508 [arXiv:1006.1311 [hep-lat]]; S. Aoki et al., Phys. Rev. D 86 (2012) 034507 [arXiv:1205.2961 [hep-lat]]; G. M. de Divitiis et al., Phys. Rev. D 87 (2013) 114505 [arXiv:1303.4896 [hep-lat]]; S. Borsanyi et al., arXiv:1306.2287 [hep-lat].

[3] M. Göckeler et al., Nucl. Phys. B 334, 527 (1990); M. Göckeler et al., Nucl. Phys. B 371 (1992) 713.

[4] N. Cundy et al., Phys. Rev. D 79 (2009) 094507 [arXiv:0901.3302 [hep-lat]].

[5] M. A. Clark and A. D. Kennedy, Phys. Rev. Lett. 98 (2007) 051601 [hep-lat/0608015].

[6] R. Horsley et al., Phys. Rev. D 85 (2012) 034506 [arXiv:1110.4971 [hep-lat]]. 